\title{
CFD Modeling and Simulation of Superheated Steam Fluidized Bed Drying Process
}

\author{
ZhiFeng Xiao ${ }^{1}$, Fan Zhang $^{1}$, NanXing Wu ${ }^{1}$, and XiangDong Liu ${ }^{2}$ \\ ${ }^{1}$ School of Mechanical and Electronic Engineering, JingDeZhen Ceramic Institute \\ JiangXi, 333403, P.R. China \\ cauxiao@126.com \\ ${ }^{2}$ College of Engineering, China Agricultural University \\ Beijing, 100083, P.R. China \\ xdliu@cau.edu.cn
}

\begin{abstract}
An unsteady mathematical model of superheated steam fluidized bed drying process is established based on the transport process principles and computational fluid dynamics (CFD) method. The vapor-solid two-phase turbulent flow in the drying chamber is described with the Eulerian-Eulerian multiphase model. The model is solved by computer numerical simulation. The drying experiments of wet rapeseeds are conducted in a normal atmosphere. The experimental results agreeing well with the simulation results show that the mathematical model of drying process is effective.
\end{abstract}

Keywords: Mathematical model, Eulerian-Eulerian, Heat and mass transfer, Rapeseed.

\section{Introduction}

Using superheated steam as the drying medium and fluidizing medium, superheated steam fluidized bed drying technology combines the advantages of superheated steam drying and fluidized bed drying to improve drying quality, reduce drying time and energy consumption by high heat and mass transfer efficiency.[1,2] In recent years, some superheated steam drying models have been developed to simulate drying wet materials but are limited by their simplifications or assumptions. Taechapairoj et al.[3] developed a drying model for granular solid, which steam flow was assumed as plug flow, and interaction between particles and steam was ignored. One-dimensional single-particle models were established and integrated with a two-phase hydrodynamic model incorporating the effects of initial condensation and superficial gas flow[4], but the sample particle was fixed and immersed in inert beads and hygroscopic porous particles that were fluidized by superheated steam under reduced pressure. The initial steam condensation rate, amount of condensed water adsorbed by particles, and

\footnotetext{
${ }^{1}$ Author: ZhiFeng Xiao, PhD, mainly engaged in Drying Theory and Computer Simulation.

D. Li and Y. Chen (Eds.): CCTA 2012, Part I, IFIP AICT 392, pp. 141-149, 2013.

(C) IFIP International Federation for Information Processing 2013
} 
water evaporation rate were considered in a model used to describe the changes in temperature and moisture content of the product with time.[5-7] A coupling model was used to simulate drying of stationary porous material by superheated steam, but the initial condensation was not considered in the whole drying process.[8] The volume shrinkage of material during drying was negligible in some models.[4,9]

Superheated steam drying is a complex process accompanied by complicated heat and mass transfer, vapor-solid interaction, and particle-particle interaction in vapor-solid two-phase turbulent flow. The above simplified models are not suitable to describe the realistic drying process. Hydrodynamic behavior of solid particles is affected by interfacial force and solid stress, which are caused by vapor-solid interaction and particle-particle interaction, respectively. The interfacial force and solid stress can be described by the Gidaspow drag model[9,10] and the well-known granular kinetic theory[11,12]. Nienwland et al.[13] studied the bubble formation in fluidized bed and found that the bubble formation, size and shape are affected by particle size, density, and minimum fluidized velocity. It is helpful to establish an mathematical model for superheated steam fluidized bed drying process.

In this paper, the wet rapeseed drying experiments are conducted using superheated steam fluidized drying technology. The CFD model is established to describe the rapeseed drying process under normal pressure.

\section{Experiments}

\subsection{Experimental Setup}

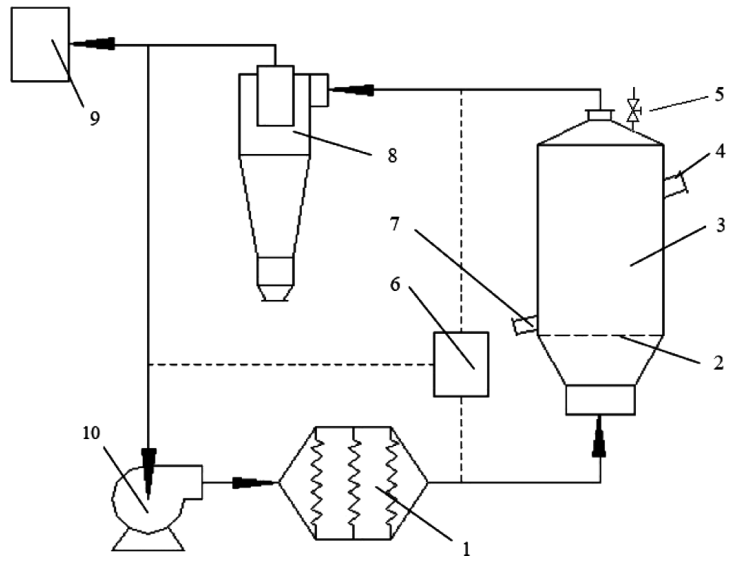

Fig. 1. Schematic diagram of the drying system

1-electric heater; 2-gas distribution orifice plate; 3-drying chamber; 4-feed inlet; 5-safety valve; 6-measurement and control system; 7-discharge outlet; 8-cyclone separator; 9-condenser; 10-blower. 
Fig. 1 presents a schematic diagram of the experimental setup for the superheated steam fluidized drying. It is a circulating system consisting of a blower, electric heater, drying chamber, condenser, measurement and control system, and other devices. The cylindrical drying chamber, whose inside diameter and height are 120 and $250 \mathrm{~mm}$ respectively, has a conical section at the lower and upper enda. An orifice plate is placed between the cylindrical drying chamber and the lower conical section; the percentage of open area is $9.8 \%$ and the diameter of the holes is $1.5 \mathrm{~mm}$. Before drying, some water is dripping into the drying chamber continuously and is heated to superheated steam by the electric heater.

\subsection{Material}

A small material is needed for the small drying chamber. Rapeseed has a small diameter and large specific surface area, is easily fluidized, and is simplified as a sphere in the drying model because it has an approximately spherical shape. Rapeseed was selected as the experimental material in the drying process.

\subsection{Experimental Procedure}

Some physical parameters of rapeseed, such as moisture content, temperature, particle density, size, and bulk porosity, are required for simulation. These parameters were measured by a moisture analyzer (MA150C-000230V1, Sartorius, Goettingen, Germany), infrared thermometer (Raynger ST6L, Raytek, Santa Cruz, CA), electronic balance (TE124S, Sartorius), oven (DHG-9140A, Jinghong, China), pycnometer, and vernier caliper. Bulk porosity can be obtained by particle density and bulk density. The normal pressure drying experiments were operated respectivly with superheated steam and hot air under the same drying conditions. The drying condition parameters (such as steam temperature, rotary velocity of blower) were set according to the experimental program, and the rapeseed was fed into the drying chamber quickly from the feed inlet. The feed inlet was closed and the rapeseeds were fluidized and dried. Sampling were performed at certain times $(0,6,45,90,135,180,225,270,315,360,405$ s) from the discharge outlet.

\section{$3 \quad$ Modeling}

\subsection{Physical Model}

The simulated object is the vapor-solid two-phase flow process and drying process in the drying chamber. To facilitate simulation, an axisymmetric cylindrical drying chamber was selected. Fig. 2 presents a schematic of the cylindrical drying chamber under a cylindrical coordinate system, in which the bottom center of the drying chamber is the origin of the coordinates. The bottom of the drying chamber is an orifice plate. Rapeseed was selected as the solid material to be dried in the small drying chamber due to its approximately spherical shape and small diameter. 


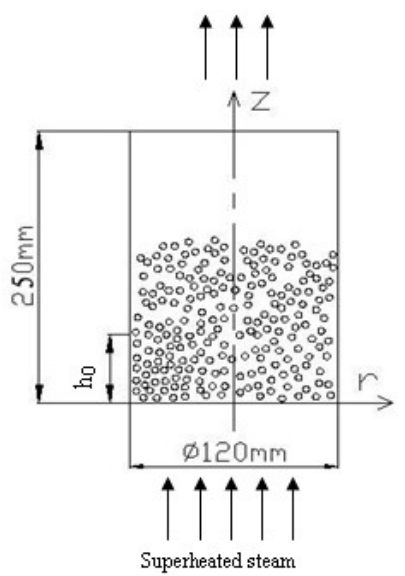

Fig. 2. Schematic of the drying chamber

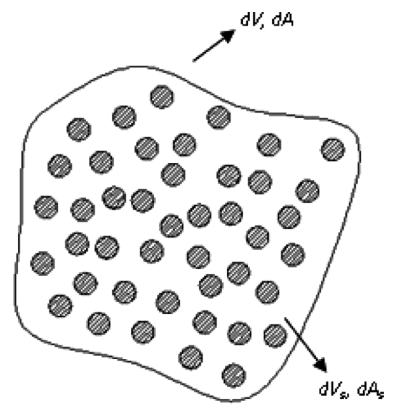

Fig. 3. Control volume of two-phase flow

\subsection{Mathematical Model}

The vapor-solid two-phase flow was treated as two-dimensional axisymmetric flow. The solid material was taken as isometric spherical particles, and the particle size was taken as constant during drying.

\section{Control volume of two-phase flow system}

The vapor-solid two-phase flow system in fluidized bed is generally viewed as a two-phase mixture, which vapor phase and solid phase occupy common space, interpenetrate and have respective properties such as dimension, velocity and temperature.[14] A control volume $(d V)$ with a surface area $(d A)$, shown in Figure 3, was chosen from the two-phase mixture in the drying chamber.

The volume fraction equation below was obeyed by at all times:

$$
a_{v}+a_{s}=1
$$

\section{Continuity equation}

The mass conservation equations are expressed as follows:

Vapor phase:

$$
\frac{\partial}{\partial t}\left(a_{v} \rho_{v}\right)+\frac{1}{r} \frac{\partial}{\partial r}\left(a_{v} \rho_{v} r u_{v r}\right)+\frac{\partial}{\partial z}\left(a_{v} \rho_{v} u_{v z}\right)=\dot{m}_{s v}
$$

Solid phase:

$$
\frac{\partial}{\partial t}\left(a_{s} \rho_{s}\right)+\frac{1}{r} \frac{\partial}{\partial r}\left(a_{s} \rho_{s} r u_{s r}\right)+\frac{\partial}{\partial z}\left(a_{s} \rho_{s} u_{s z}\right)=\dot{m}_{v s}
$$

The moisture mass fraction of granule $X^{\prime}$, can be predicted by Equation (4): 


$$
\frac{\partial}{\partial t}\left(a_{s} \rho_{s} X^{\prime}\right)+\frac{1}{r} \frac{\partial}{\partial r}\left(a_{s} \rho_{s} r u_{s r} X^{\prime}\right)+\frac{\partial}{\partial z}\left(a_{s} \rho_{s} u_{s z} X^{\prime}\right)=\nabla \cdot\left(a_{s} \rho_{s} D_{w, s} \nabla X^{\prime}\right)+\dot{m}_{v s}
$$

\section{Momentum conservation equations}

Steam phase:

$$
\frac{\partial}{\partial t}\left(\alpha_{v} \rho_{v} \vec{u}_{v}\right)+\nabla \cdot\left(\alpha_{v} \rho_{v} \vec{u}_{v} \vec{u}_{v}\right)=-\alpha_{v} \nabla P+\nabla \cdot \overline{\bar{\tau}}_{v}+a_{v} \rho_{v} \vec{g}+\vec{R}_{v s}+\dot{m}_{s v} \vec{u}_{s v}
$$

Solid phase:

$$
\frac{\partial}{\partial t}\left(\alpha_{s} \rho_{s} \vec{u}_{s}\right)+\nabla \cdot\left(\alpha_{s} \rho_{s} \vec{u}_{s} \vec{u}_{s}\right)=-\alpha_{s} \nabla P-\nabla P_{s}+\nabla \cdot \overline{\bar{\tau}}_{s}+a_{s} \rho_{s} \vec{g}+\vec{R}_{s v}+\dot{m}_{v s} \vec{u}_{v s}
$$

$\vec{R}_{v s}$ and $\vec{R}_{s v}$ are drag forces between the two phases, which are defined as follows.

\section{Energy conservation equations}

The energy conservation equations of two-phase flow are formulated as follows:

Vapor phase:

$$
\frac{\partial}{\partial t}\left(\alpha_{v} \rho_{v} H_{v}\right)+\nabla \cdot\left(\alpha_{v} \rho_{v} \vec{u}_{v} H_{v}\right)=-\alpha_{v} \frac{\partial P}{\partial t}+\overline{\bar{\tau}}_{v}: \nabla \vec{u}_{v}+Q_{s v}+\dot{m}_{s v} H_{e v p}
$$

Solid phase:

$$
\frac{\partial}{\partial t}\left(\alpha_{s} \rho_{s} H_{s}\right)+\nabla \cdot\left(\alpha_{s} \rho_{s} \vec{u}_{s} H_{s}\right)=-\alpha_{s} \frac{\partial P}{\partial t}+\overline{\bar{\tau}}_{s}: \nabla \vec{u}_{s}+Q_{v s}+\dot{m}_{v s} H_{e v p}
$$

\section{Heat and mass transfer model}

The drying process can be divided into three distinct periods: the condensing and heating period, constant drying rate period, and falling drying rate period.

Condensing and heating period $\left(T_{\mathrm{s} 0} \leq T_{\mathrm{s}}<T_{\mathrm{b}}\right)$ :

The condensation convective heat transfer coefficient is determined according to the method of McAdams[15]:

$$
h_{c}=1.13 \times \frac{6 \alpha_{s}}{d_{s}} \times\left(\frac{\left[H_{e v p}+C_{p v}\left(T_{v}-T_{b}\right)\right] \rho_{l}^{2} g \lambda_{l}^{3}}{\mu_{l} d_{s}\left(T_{v}-T_{b}\right)}\right)^{1 / 4}
$$

The mass transfer rate between steam and particles:

$$
\dot{m}_{v s}=\frac{Q_{s v}}{H_{e v p}+C_{p v}\left(T_{v}-T_{b}\right)}
$$

Where

$$
Q_{s v}=h_{c}\left(T_{b}-T_{v}\right)
$$


Constant drying rate period $\left(T_{\mathrm{s}}=T_{\mathrm{b}}, X \geq X_{\mathrm{cr}}\right)$ :

The convective heat transfer rate:

$$
h=\frac{6 \lambda_{v} a_{s} N u_{s}}{d_{s}^{2}}
$$

Where

$$
N u_{s}=2.0+0.74 \operatorname{Re}_{s}^{\frac{1}{2}} \operatorname{Pr}^{\frac{1}{3}}
$$

The mass transfer rate:

$$
\dot{m}_{s v}=\frac{Q_{v s}}{H_{e v p}}
$$

Where

$$
Q_{v s}=h\left(T_{v}-T_{s}\right)
$$

Falling drying rate period $\left(T_{\mathrm{b}}<T_{\mathrm{s}} \leq T_{\mathrm{v} 0}, X_{\mathrm{eq}} \leq X<X_{\mathrm{cr}}\right)$ :

The mass transfer rate in particles:

$$
\dot{m}_{s v}=-\alpha_{s} \rho_{s} \frac{d \bar{X}}{d t}=\frac{6 \alpha_{s} \rho_{s} D_{\mathrm{eff}}\left(X_{c r}-X_{e q}\right)}{d_{s}^{2}} \exp \left(-4 \pi^{2} \frac{D_{\mathrm{eff}}\left(t-t_{\mathrm{cr}}\right)}{d_{s}^{2}}\right)
$$

The heat transfer rate:

$$
Q_{v s}=h\left(T_{v}-T_{s}\right)=\alpha_{s} \rho_{s} C_{p s} \frac{d T_{s}}{d t}+\dot{m}_{s v} H_{e v p}
$$

\section{Numerical solution}

The finite volume method was introduced to numerically solve the drying model using FLUENT. Furthermore, the heat and mass transfer model, drag force model, and physical parametric model were programmed using $\mathrm{C}$ and then incorporated into FLUENT through a user-defined function (UDF) to solve the drying model.

\section{$4 \quad$ Results and Discussion}

\subsection{Drying Condition Parameters and Equipment Parameters}

The inlet velocity of superheated steam was selected as $2.5 \mathrm{~m} \cdot \mathrm{s}^{-1}$ during the drying experiments. Some parameters used in the experiments are shown in Table 1. 
Table 1. Drying condition parameters and equipment parameters

\begin{tabular}{lll}
\hline \multicolumn{1}{c}{ Parameters } & \multicolumn{1}{c}{ Unit } & \multicolumn{1}{c}{ Value } \\
\hline Steam pressure & $\mathrm{Pa}$ & $1.01 \times 10^{5}$ \\
Environmental temperature & $\mathrm{K}$ & 300.2 \\
Inlet steam temperature & $\mathrm{K}$ & 423 \\
Initial moisture content of rapesed $(\mathrm{db})$ & $\mathrm{kg}^{-1} \mathrm{~kg}^{-1}$ & 0.433 \\
Initial particle density of rapeseed & $\mathrm{kgm}^{-3}$ & 1184 \\
Bone dry particle density of rapaseed & $\mathrm{kgm}^{-3}$ & 817 \\
Mean particle size of rapeseed & $\mathrm{mm}$ & 1.76 \\
Depth of static rapseed bed & $\mathrm{mm}$ & 80 \\
Porosity of papeseed in the static bed & - & 0.43 \\
Size of drying chamber & $\mathrm{mm}$ & $250 \times \phi 120$ \\
Rotary velocity of fan & $\mathrm{r} \mathrm{min}$ & 1840 \\
Rated power of electric heated & $\mathrm{kW}$ & 9 \\
\hline
\end{tabular}

\subsection{Drying Dynamic and Simulation Results}

Figure 4 shows the simulation and experimental results for moisture content of rapeseed. Figure 5 shows the drying rate curves from simulation and experiment. The whole drying process can be divided into three periods: the condensing and heating period, constant drying rate period, and falling drying rate period. The simulated drying curve is in agreement with the experimental results in three periods.

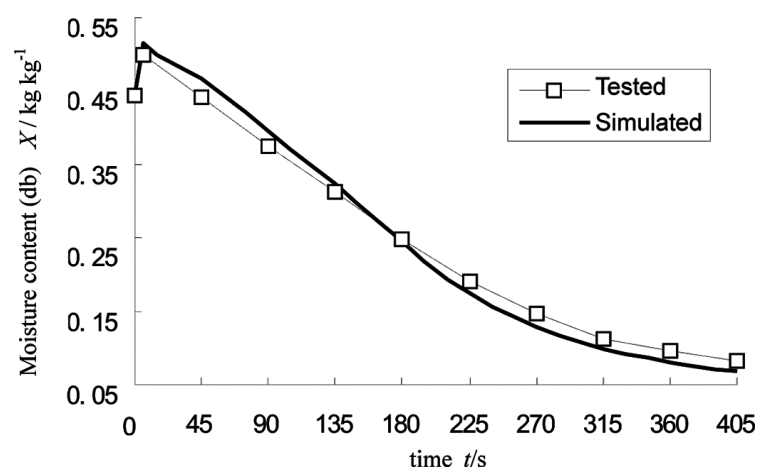

Fig. 4. Moisture content of rapeseed from simulation and experiment 


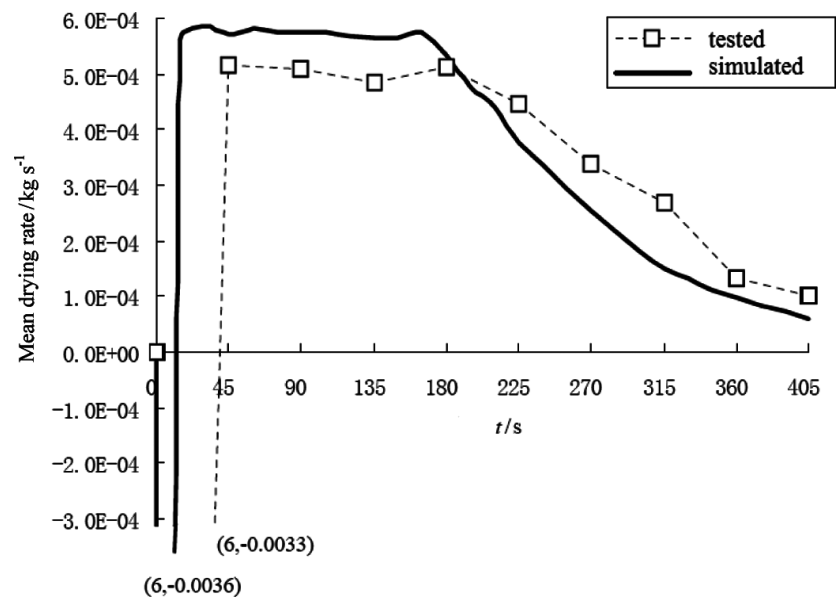

Fig. 5. Drying rate curve of rapeseed from simulation and experiment

A certain difference was found between the simulated drying curve and the experimental curve at the beginning. The simulated moisture content was greater than the experimental result in this period because the simulated curve ascends faster than latter. This discrepancy may be due to the fact that only steam condensation was taken into account and water evaporation from the material was ignored in simulation, though water evaporation from the material occurred during steam condensation. During the constant drying rate period, the simulated moisture content decreased faster; that is, the simulated drying rate was greater than that during the experiment.

\section{Conclusions}

The simulated drying curve obtained from the drying model was in good agreement with the experimental results. A negative drying rate occurred in the first period caused by initial steam condensation and lasted for about $6 \mathrm{~s}$, and the moisture content of material increased up to a maximum. In the constant drying rate period, the simulated constant drying rate was greater than that of the experiment, which resulted in a shorter constant drying rate period. The simulated drying rate curve declined faster and a higher material temperature was obtained from simulation in the falling drying rate period.

\section{References}

1. Kudra, T., Mujumdar, A.S.: Advanced Drying Technologies. Marcel Dekker Inc., New York (2002)

2. Xiao, Z.F.: Numerical Simulation and Experimental Study on Fluidized Bed Drying Process with Superheated Steam. China Agricultural University Doctoral Thesis, Beijng (2008) 
3. Taechapairoj, C., Prachayawarakorn, S., Soponronnarit, S.: Modelling of parboiled rice in superheated steam fluidized bed. Journal of Food Engineering 76(3), 411-419 (2006)

4. Tatemoto, Y., Yanoa, S., Mawatarib, Y., Nodaa, K., Komatsuc, N.: Drying characteristics of porous material immersed in a bed of glass beads fluidized by superheated steam under reduced pressure. Chemical Engineering Science 62(1-2), 471-480 (2007)

5. Soponronnarit, S., Prachayawarakorn, S., Rordprapat, W., Nathakaranakule, A., Tia, W.: A superheated steam fluidized bed dryer for parboiled rice: Testing of a pilot-scale and mathematical model development. Drying Technology 24(11), 1457-1467 (2006)

6. Poomjai, S., Thanit, S., Adisak, N., Somchart, S.: Mathematical model of pork slice drying using superheated steam. Journal of Food Engineering 104, 499-507 (2011)

7. Pakowski, Z., Adamski, R.: On prediction of the drying rate in superheated steam drying process. Drying Technology 29, 1492-1498 (2011)

8. Erriguible, A., Bernada, P., Couture, F., Roques, M.: Simulation of superheated steam drying from coupling model. Drying Technology 24(8), 941-951 (2006)

9. Wu, Z.H., Mujumdar, A.S.: Simulation of the hydrodynamics and drying in a spouted bed dryer. Drying Technology 25(1), 59-74 (2007)

10. Gidaspow, D., Bezburuah, R., Ding, J.: Hydrodynamics of circulating fluidized beds, kinetic theory approach. In: Fluidization VII, Proceedings of the 7th Engineering Foundation Conference on Fluidization, Brisbane, Australia (1992)

11. Du, W., Bao, X.J., Xu, J., Wei, W.S.: Computational fluid dynamics(CFD) modeling of spouted bed: Assessment of drag coefficient correlations. Chemical Engineering Science 61(5), 1401-1420 (2006)

12. Neri, A., Gidaspow, D.: Riser hydrodynamics: simulation using kinetic theory. AIChE Journal 46(1), 52-67 (2000)

13. Nienwland, J.J., Veenendaal, M.L., Kuipers, J.A.M., van Swaaij, W.P.M.: Bubble formation at a single orifice in gas fluidized beds. Chemical Engineering Science 51(17), 4087-4102 (1996)

14. Zhou, L.X.: Theory and Numerical Modeling of Turbulent Gas-Particle Flows and Combustion. Science Publishing Co., Beijing (1994)

15. Tao, W.Q.: Numerical Heat Transfer, 2nd edn. Xi'an Jiaotong University Press, Xi'an (2001) 